\title{
Relativistic Quantum Motion of an Electron in Spinning Cosmic String Spacetime in the Presence of Uniform Magnetic Field and Aharonov-Bohm Potential
}

\author{
Márcio M. Cunha $(\mathbb{D}$ and Edilberto O. Silva \\ Departamento de Física, Universidade Federal do Maranhão, 65085-580 São Luís, Maranhão, Brazil \\ Correspondence should be addressed to Edilberto O. Silva; edilbertoo@gmail.com
}

Received 2 June 2021; Accepted 30 October 2021; Published 26 November 2021

Academic Editor: Elias C. Vagenas

Copyright (C) 2021 Márcio M. Cunha and Edilberto O. Silva. This is an open access article distributed under the Creative Commons Attribution License, which permits unrestricted use, distribution, and reproduction in any medium, provided the original work is properly cited. The publication of this article was funded by SCOAP . $^{3}$

\begin{abstract}
In this manuscript, we study the relativistic quantum mechanics of an electron in external fields in the spinning cosmic string spacetime. We obtain the Dirac equation and write the first- and second-order equations from it, and then, we solve these equations for bound states. We show that there are bound state solutions for the first-order equation Dirac. For the secondorder equation, we obtain the corresponding wave functions, which depend on the Kummer functions. Then, we determine the energies of the particle. We examine the behavior of the energies as a function of the physical parameters of the model, such as rotation, curvature, magnetic field, Aharonov-Bohm flux, and quantum numbers. We find that, depending on the values of these parameters, there are energy nonpermissible levels.
\end{abstract}

\section{Introduction}

Symmetry is key ingredient in the description of natural phenomena. The notion of symmetry is an essential feature in several areas of physics. In this context, the well-known Noether's theorem [1] establishes a connection between symmetry and conservation laws of relevant physical quantities. In quantum mechanics, we often use symmetry to obtain crucial results concerning angular momenta operators [2]. Likewise, symmetry is relevant in the topic of quantum information [3]. Also, symmetry is essential in the framework of relativity [4], and for this reason, it is indispensable in research areas such as particle physics [5] and cosmology [6].

A pertinent question in the research areas cited above refers to think about the consequences of symmetrybreaking in a given physical system. It can happen in several contexts, like in phase transitions, for example [7-9]. Another example of symmetry-breaking occurs in solids: we can create topological defects like disclinations and dislocations $[10,11]$ from the Volterra process [12].

Topological defects can emerge in a large number of physical systems covering themes such as liquid crystals
[13], graphene physics [14, 15], magnetism [16], and cosmology [17]. Recent studies also have reported the importance of topological defects in Life Sciences $[18,19]$. In cosmology, defects in the spacetime topology can be viewed as a possible consequence of the evolution of the early universe, which has suffered phase transitions due to the temperature decreasing and the process of expansion $[20,21]$.

In this contribution, we are involved in studying the topological defect known as a cosmic string. A cosmic string is a linear defect, similar to a flux tube in type-II superconductors [20]. The spacetime around such defect has a conical symmetry, being identical to the case of a disclination [22]. The concept of a cosmic string was introduced in the literature by Kibble [23]. An intriguing facet in this subject refers to the quantum mechanical description of a particle in a region of the spacetime containing this defect. There are several works dealing with this issue, both in the nonrelativistic and relativistic scenarios. Reference [24], for instance, analyzes the hydrogen atom in a spacetime of a cosmic string. Reference [25] considers the problem of a relativistic electron in the presence of both Coulomb and scalar potentials in the cosmic string spacetime. Results about vacuum 
polarization in a cosmic string spacetime were reported in Ref. [26]. Again, the cosmic string spacetime was considered as a background to examine relativistic oscillators [27-30], quantum phases [31], and fermionic currents [32].

A relevant issue in this context consists of taking into consideration the influence of electromagnetic fields in the quantum particle motion. Landau levels [33] and the Aharonov-Bohm effect $[34,35]$, for instance, are essential ingredients in the investigation of quantum systems even in a flat spacetime. Landau levels are a quantum analog of classical cyclotron motion, while the Aharonov-Bohm effect reveals the significance of the vector potential in the quantum world. Then, studying the contribution of these magnetic interactions to the quantum mechanical description of a system in spacetime having a topological defect is a natural development. In Ref. [36], for example, it was considered the interaction of a point charge with a magnetic field in a spacetime with a distortion. References [37-44] are examples of studies dealing with Landau levels and the Aharonov-Bohm effect in the presence of topological defects. Reference [45] deals with a quantum ring in graphene with a topological defect and a magnetic field. Reference [46] investigates an analog of the Aharonov-Bohm effect for bound states in a spacetime containing a spacelike dislocation. In particular, the inclusion of electromagnetic interactions in the case of a cosmic string background also has been considered. For instance, Ref. [47] analyzes the quantum dynamics of a charged particle in the presence of a magnetic field and scalar potential. Reference [48] examines different configurations of confined magnetic fields and investigates the existence of induced vacuum fermionic currents.

On the other hand, we can be interested in analyzing the behavior of rotating systems. It is a pertinent question, since large structures in the Universe, such as black holes and galaxies, have angular momentum due to rotation [49]. Also, it is possible to conceive cosmologic models with rotation [50]. The study of quantum systems in spinning spacetimes also has been reported in the literature [51-53]. Noninertial effects in quantum systems can provide novel theoretical predictions and feasible experimental developments. For instance, quantum phases in rotating systems can emerge, in analogy to the Aharonov-Bohm effect $[54,55]$. It is also possible to establish a relation between the Hall effect and the inertial forces [56]. Besides, if a given system is rotating, physical properties like spin transport $[57,58]$ and electronic structure [59] are modified. A system can present magnetization due to rotation, like in the Barnett effect [60]. While a magnetic field produces a spin-field coupling, resulting in the anomalous Zeeman effect [61], rotation produces an analog effect, due to the spin-rotation coupling [62]. Thus, rotation can contribute similarly to a magnetic field in the dynamics of a quantum system. More, noninertial effects are an interesting issue when the spacetime contains topological defects. In this case, we can include noninertial effects and the topological defects in the quantum mechanical description by employing the same tool. More specifically, we can use a metric tensor to a spinning spacetime with a topological defect [63]. The spacetime of a spinning cosmic string has been considered as background for several problems involving quantum systems [64]. For instance, Ref. [65] deals with the Schrödinger equation in that spacetime. Reference [66] analyzed the bound states for neutral particles in a rotating frame of a cosmic string. Likewise, Ref. [67] investigates rotating effects on a Landau-AharonovCasher System in the spacetime of a cosmic string.

As we already have mentioned, rotation can present similarities to the electromagnetic fields. This way, it is also an attractive question examining how the electromagnetic interactions affect the particle quantum motion of a rotating system in the presence of a topological defect. Reference [68], for instance, is a recent work dealing with both topological and noninertial effects in the presence of an Aharonov-Bohm potential. Reference [69] addresses the problem of a spinless relativistic particle subjected to a uniform magnetic field in the spinning cosmic string spacetime. The Dirac oscillator in the spacetime of a cosmic string in the presence of the Aharonov-Casher effect was analyzed in Ref. [70]. The presence of noninertial effects also was considered in this context [71]. Reference [72] treats the problem of a charged half-spin particle depicted by the Dirac equation in the presence of a uniform magnetic field in the rotating cosmic string spacetime. A meaningful aspect in this context consists of analyzing how different configurations of magnetic fields affect the quantum particle motion. In particular, in this contribution, we choose to deal with a superposition of two different configurations, due to their fundamental aspects: a uniform magnetic field, which is related to the raising of Landau levels in quantum systems, and an Aharonov-Bohm flux, since it provides a new significance to the role of the electromagnetic interactions in the quantum theory, which can be manifested even for bound states [73]. Besides, several analogs of the $\mathrm{AB}$ effect can emerge in spacetimes with topological defects [74-76].

In this paper, we study the relativistic quantum mechanics of an electron in the presence of both a uniform magnetic field and Aharonov-Bohm potential in the spinning cosmic string spacetime. In other words, we solve the Dirac equation in this scenario and investigate how the rotation, curvature, and external magnetic fields affect the wave functions and energies of the electron. Although the existence of a previous study deals with the Dirac equation in the presence of noninertial effects and uniform magnetic field, in Section 3 of Ref. [72], here, we introduce some new ingredients, as well as a different framework: more explicitly, we have the following contributions: (i) the tetrad basis we adopt leads to a zero affine connection in the flat space, which does not occur in the previous study; (ii) the presence of the Aharonov-Bohm flux tube. We show explicitly that the presence of this effect modifies the energy levels of the particle; (iii) we also study first-order solutions for the Dirac equation and find particular bound state solutions for it; (iv) we employ an ansatz for the solutions whose spinor is an eigenstate of the momentum angular operator $\bar{J}^{\text {TmqZgTqqhF-; }} z$ [47].

The manuscript is organized as follows. In Section 2, we present some algebraic elements necessary to construct the 
field equations in curved spacetime and write the Dirac equation describing the quantum motion of the electron in the presence of external magnetic fields in the spinning cosmic string background. In Section 3, we deal with first-order solutions and study the existence of isolated solutions for the particular case of a particle at rest. In Section 4, we take our attention to the case when the energy of the particle is different from its rest energy. We map the Dirac equation problem in curved space with minimal coupling into a SturmLiouville problem for the upper component of the Dirac spinor, and using an appropriate ansatz, we derive the radial equation. We solve the radial equation and find the wave functions and energies of the particle. We make a detailed discussion of the results and also comparisons with other studies in the literature. In Section 5, we present our conclusions. In our work, we use natural units, $\hbar=c=G=1$.

\section{Dirac Equation in the Spinning Cosmic String Spacetime}

In this section, we briefly present the tools needed to construct the Dirac equation in the conical spacetime in the presence of noninertial effects. The first step consists of taking a look at the metric tensor characterizing this geometry. Next, we will choose an appropriate tetrad basis and implement the field configuration involved through the performing of a minimal substitution. The spacetime induced by a rotating cosmic string is described by the metric $[72,77,78]$.

$$
d s^{2}=(d t+a d \varphi)^{2}-d r^{2}-\alpha^{2} r^{2} d \varphi^{2}-d z^{2}
$$

where $-\infty<z<\infty, r \geq 0$, and $0 \leq \varphi \leq 2 \pi$. The parameter $\alpha$ is related to the linear mass density $\mu$ of the cosmic string through the relation $\alpha=1-4 \mu$, and it runs in the interval $(0,1]$. The quantity $a=4 J$ is the rotation parameter, with $J$ representing the angular momentum of the spinning cosmic string. A remarkable feature of the spinning cosmic string spacetime is related to the appearance of closed timelike curves (CTCs) [79-81]. Such structures can take place depending on the relation between the radial coordinate, the rotation parameter, and the curvature parameter. Writing the metric (1) in the form

$$
d s^{2}=d t^{2}+2 a d t d \varphi-\left(\alpha^{2} r^{2}-a^{2}\right) d \varphi^{2}-d r^{2}-d z^{2}
$$

such a structure appears if $\alpha^{2} r^{2}-a^{2}<0$. CTCs are one of the most controversial aspects of modern physics [82]. It is possible to find in the literature works dealing with the concepts of causality [83], chronology protection [84], time travel [85], and flow information [86] in the framework of closed timelike curves. In our study, we consider the quantum motion only in regions such that $\alpha^{2} r^{2}-a^{2}>0$. More explicitly, we will consider the following restriction on the radial coordinate:

$$
r>\frac{a}{\alpha}
$$

This type of restriction on the radial coordinate also occurs, for example, in the case of a spacetime containing a time dislocation, as pointed in Ref. [51].

The relativistic quantum dynamics of a spin- $1 / 2$ particle interacting with external magnetic fields in the rotating cosmic string spacetime is governed by the Dirac equation

$$
\left[i \gamma^{\mu}(x)\left(\nabla_{\mu}+i e A_{\mu}(x)\right)-M\right] \Psi(x)=0,
$$

where $M$ is the mass of the particle and $\nabla_{\mu}$ is the covariant derivative for fermion fields defined as

$$
\nabla_{\mu}=\partial_{\mu}+\Gamma_{\mu}(x)
$$

and $\gamma^{\mu}(x)$ are the Dirac matrices in the rotating cosmic string spacetime, which are defined in terms of the tetrad fields $e_{a}^{\mu}$ and Dirac matrices in the flat space $\gamma^{a}$ in the following way:

$$
\gamma^{\mu}(x)=e_{a}^{\mu}(x) \gamma^{a}
$$

where

$$
\gamma^{a}=\left(\gamma^{0}, \gamma^{i}\right) \text {, with } \gamma^{0}=\left(\begin{array}{cc}
1 & 0 \\
0 & -1
\end{array}\right), \gamma^{i}=\left(\begin{array}{cc}
0 & \sigma^{i} \\
-\sigma^{i} & 0
\end{array}\right) \text {, }
$$

are the standard Dirac matrices and $\sigma^{i}=\left(\sigma^{x}, \sigma^{y}, \sigma^{z}\right)$ are the usual Pauli matrices. The matrices of (6) satisfy the following relation:

$$
\left\{\gamma^{\mu}(x), \gamma^{v}(x)\right\}=2 g^{\mu \nu}(x)
$$

Also, in Equation (5), $\Gamma_{\mu}(x)$ is the spin affine connection given by

$$
\Gamma_{\mu}(x)=\frac{1}{4} \gamma^{a} \gamma^{b} e_{a}^{v}(x)\left[\partial_{\mu} e_{b v}(x)-\Gamma_{\mu \nu}^{\sigma} e_{b \sigma}(x)\right]
$$

where $\Gamma_{\mu \nu}^{\sigma}$ are the Christoffel symbols of the second kind and $e_{a}^{\mu}(x)$ is the tetrad field. The tetrad basis satisfies the relations

$$
\begin{gathered}
e_{\mu}^{a}(x) e_{v}^{b}(x) \eta_{a b}=g_{\mu \nu}(x), \\
e_{\mu}^{a}(x) e_{b}^{\mu}(x)=\delta_{b}^{a}, \\
e_{a}^{\mu}(x) e_{v}^{a}(x)=\delta_{v}^{\mu} .
\end{gathered}
$$


Here, the Greek letters are used for tensor indices while the Latin letters are denoting Minkowski indices. We use the tetrad basis and its inverse defined as [77]

$$
\begin{gathered}
e_{\mu}^{a}(x)=\left(\begin{array}{cccc}
1 & 0 & a & 0 \\
0 & \cos \varphi & -r \alpha \sin \varphi & 0 \\
0 & \sin \varphi & r \alpha \cos \varphi & 0 \\
0 & 0 & 0 & 1
\end{array}\right), \\
e_{a}^{\mu}(x)=\left(\begin{array}{cccc}
1 & \frac{a \sin \varphi}{r \alpha} & -\frac{a \cos \varphi}{r \alpha} & 0 \\
0 & \cos \varphi & \sin \varphi & 0 \\
0 & -\frac{\sin \varphi}{r \alpha} & \frac{\cos \varphi}{r \alpha} & 0 \\
0 & 0 & 0 & 1
\end{array}\right) .
\end{gathered}
$$

For this choice, it can be shown that the nonvanishing affine connection is given by

$$
\Gamma_{\mu}=\left(0,0, \Gamma_{\varphi}, 0\right), \text { with } \Gamma_{\varphi}=\frac{i}{2}(1-\alpha) \sum^{z},
$$

where

$$
\sum^{z}=\left(\begin{array}{cc}
\sigma^{z} & 0 \\
0 & \sigma^{z}
\end{array}\right), \sigma^{z}=\left(\begin{array}{cc}
1 & 0 \\
0 & -1
\end{array}\right) .
$$

By using the tetrad basis (14), the matrices of (6) can be written explicitly as

$$
\begin{gathered}
\gamma^{t}=\gamma^{0}-a \gamma^{\varphi}, \gamma^{0}=\left(\begin{array}{cc}
1 & 0 \\
0 & -1
\end{array}\right), \\
\gamma^{r}=\left(\begin{array}{cc}
0 & \sigma^{r} \\
-\sigma^{r} & 0
\end{array}\right), \gamma^{\varphi}=\left(\begin{array}{cc}
0 & \sigma^{\varphi} \\
-\sigma^{\varphi} & 0
\end{array}\right),
\end{gathered}
$$

with

$$
\sigma^{r}=\left(\begin{array}{cc}
0 & e^{-i \varphi} \\
e^{+i \varphi} & 0
\end{array}\right), \sigma^{\varphi}=\frac{1}{r \alpha}\left(\begin{array}{cc}
0 & -i e^{-i \varphi} \\
i e^{+i \varphi} & 0
\end{array}\right)
$$

being the Pauli matrices in the curved spacetime.

Since we are first interested in studying the solutions of the Dirac equation in its present form (Equation (4)), we need to write the corresponding system of first-order coupled differential equations. Let us assume the timedependence of the wave functions together with the decomposition of the fermion field in the form

$$
\Psi(r, \varphi)=e^{-i E t}\left(\begin{array}{l}
\psi_{1}(r, \varphi) \\
\psi_{2}(r, \varphi)
\end{array}\right),
$$

with

$$
\begin{gathered}
\psi_{1}(r, \varphi)=\left(\begin{array}{c}
\psi_{a}(r, \varphi) \\
\psi_{b}(r, \varphi)
\end{array}\right)=\left(\begin{array}{c}
e^{i m \varphi} f_{+}(r) \\
i e^{i(m+1) \varphi} f_{-}(r)
\end{array}\right), \\
\psi_{2}(r, \varphi)=\left(\begin{array}{c}
\psi_{c}(r, \varphi) \\
\psi_{d}(r, \varphi)
\end{array}\right)=\left(\begin{array}{c}
e^{i m \varphi} g_{+}(r) \\
i e^{i(m+1) \varphi} g_{-}(r)
\end{array}\right) .
\end{gathered}
$$

The system we will analyze takes into account the particle is immersed in a region where there is a uniform magnetic field and also the potential due to a thin long solenoid along the $z$-axis. Having this field configuration in mind, we study the physical implications due to noninertial effects and the Aharonov-Bohm potential on the relativistic Landau quantization. We also take into account the translational invariance of the system along the $z$-direction, which allows us to eliminate the third direction $\left(p_{z}=z=0\right)$, and, consequently, we can consider only the planar motion [87-90]. Here, the four-potential of the electromagnetic field in Equation (4) has only the spatial component, $A_{\mu}=(0, \mathbf{A})$, and the potential vector in the Coulomb gauge is specified by

$$
\mathbf{A}=\left(0, A_{\varphi}, 0\right)
$$

with

$$
\begin{gathered}
A_{\varphi}=-\left(A_{\varphi, 1}+A_{\varphi, 2}\right), \\
A_{\varphi, 1}=\frac{1}{2} \alpha B r^{2}, A_{\varphi, 2}=\frac{\phi}{e} \frac{1}{\alpha r},
\end{gathered}
$$

where $B$ is the magnetic field magnitude, $\phi=\Phi / \Phi_{0}, \Phi$ is the magnetic flux, and $\Phi_{0}=2 \pi / e$ is the quantum of magnetic flux along the solenoid. This configuration provides an superposition of magnetic fields in the $z$-direction

$$
B=B_{z, 1}+B_{z, 2}
$$

with

$$
B_{1, z}=B, B_{z, 2}=\frac{\phi}{e} \frac{\delta(r)}{\alpha r}
$$

Note that the particle only interacts with the magnetic field due to the potential vector $A_{\varphi, 1}$. Here, we are focused on studying the electron motion only in the $r \neq 0$ region, so that we can neglect the point interaction $B_{z, 2}$ and, consequently, consider only regular wave functions.

Using the results above, the Dirac equation (4) can be written as

$$
(E-M) \psi_{1}+\sigma^{r} i \partial_{r} \psi_{2}+\sigma^{\varphi}\left(i \partial_{\varphi}-e A_{\varphi}-a E-\frac{1}{2}(1-\alpha) \sigma^{z}\right) \psi_{2}=0,
$$


$(E+M) \psi_{2}+\sigma^{r} i \partial_{r} \psi_{1}+\sigma^{\varphi}\left(i \partial_{\varphi}-e A_{\varphi}-a E-\frac{1}{2}(1-\alpha) \sigma^{z}\right) \psi_{1}=0$

At this point, we are ready to solve Equations (28) and (29) by considering two distinct circumstances:

(i) Take our attention to isolated solutions of the firstorder Dirac equation by imposing the condition $E$ $= \pm M$

(ii) By imposing the condition $E \neq \pm M$, we search the solutions of the second-order Dirac equation

We will show in the next two sections that there are bound state solutions for both cases and discuss their main physical properties. To distinguish each case in (i), in the next section, we use the superscripts $( \pm)$ to label the quantities corresponding to $E= \pm M$.

3. Solution of the Equation of Motion to $E= \pm M$

To study the existence of isolated solutions of the Dirac equation (4), we must set $E= \pm M$ in Equations (28) and (29). In literature, such solutions are known to be excluded from the Sturm-Liouville problem. The search for isolated solutions of the Dirac equation has been performed in different physical contexts [91-95]. Since condition (3) establishes a lower limit to the radial coordinate, given by $r_{\min }=a / \alpha$, the bound state solution must satisfy the normalization condition

$$
\int_{r_{\min }}^{\infty} \bar{\Psi} \gamma^{t} \Psi r d r=\int_{r_{\min }}^{\infty} \Psi^{\dagger} \Psi r d r-a \int_{r_{\min }}^{\infty} \Psi^{\dagger} \gamma^{0} \gamma^{\varphi} \Psi r d r
$$

where $\bar{\Psi}=\Psi^{\dagger} \gamma^{0}$, which using the ansatz (20) and the matrices (18) and (17), takes the form

$$
\begin{gathered}
\int_{r_{\min }}^{\infty}\left[\left|f_{+}(r)\right|^{2}+\left|f_{-}(r)\right|^{2}+\left|g_{+}(r)\right|^{2}+\left|g_{-}(r)\right|^{2}\right] r d r \\
-\frac{a}{\alpha} \int_{r_{\min }}^{\infty}\left[f_{+}^{\dagger}(r) g_{-}(r)+f_{-}^{\dagger}(r) g_{+}(r)\right. \\
\left.+g_{+}^{\dagger}(r) f_{-}(r)+g_{-}^{\dagger}(r) f_{+}(r)\right] d r .
\end{gathered}
$$

Equations (30) and (31) must be finite to ensure the existence of bound state solutions. By making $E=+M$ in Equations (28) and (29) and using Equations (21) and (22), we get

$$
\begin{aligned}
e^{i \varphi} i \partial_{\mathrm{r}} \psi_{c}(r, \varphi) & +\frac{i}{r \alpha} e^{i \varphi}\left(i \partial_{\varphi}-e A_{\varphi}-a M\right) \psi_{c}(r, \varphi) \\
& -\frac{i}{2 r \alpha}(1-\alpha) e^{i \varphi} \psi_{c}(r, \varphi)=0,
\end{aligned}
$$

$$
\begin{aligned}
e^{-i \varphi} i \partial_{r} \psi_{d}(r, \varphi) & -\frac{i}{r \alpha} e^{-i \varphi}\left(i \partial_{\varphi}-e A_{\varphi}-a M\right) \psi_{d}(r, \varphi) \\
& -\frac{i}{2 r \alpha}(1-\alpha) e^{-i \varphi} \psi_{d}(r, \varphi)=0 \\
i \partial_{r} e^{i \varphi} \psi_{a}(r, \varphi) & +\frac{i}{r \alpha} e^{i \varphi}\left(i \partial_{\varphi}-e A_{\varphi}-a M\right) \psi_{a}(r, \varphi) \\
& -\frac{i}{2 \alpha r}(1-\alpha) e^{i \varphi} \psi_{a}=-2 M \psi_{d}(r, \varphi) \\
i \partial_{r} e^{-i \varphi} \psi_{b}(r, \varphi) & -\frac{i}{r \alpha} e^{-i \varphi}\left(i \partial_{\varphi}-e A_{\varphi}-a M\right) \psi_{b}(r, \varphi) \\
& -\frac{i}{2 \alpha r}(1-\alpha) e^{-i \varphi} \psi_{b}(r, \varphi)=-2 M \psi_{c}(r, \varphi)
\end{aligned}
$$

which using Equation (25) can be written as

$$
\begin{gathered}
\frac{d g_{+}^{(+)}(r)}{d r}-\frac{L_{m}^{(+)}}{r \alpha} g_{+}^{(+)}(r)+\frac{e B r}{2} g_{+}^{(+)}(r)=0 \\
\frac{d g_{-}^{(+)}(r)}{d r}+\frac{L_{m+1}^{(+)}}{r \alpha} g_{-}^{(+)}(r)-\frac{e B r}{2} g_{-}^{(+)}(r)=0 \\
\frac{d f_{+}^{(+)}(r)}{d r}-\frac{L_{m}^{(+)}}{r \alpha} f_{+}^{(+)}(r)+\frac{e B r}{2} f_{+}^{(+)}(r)=-2 M g_{-}^{(+)}(r), \\
\frac{d f_{-}^{(+)}(r)}{d r}+\frac{L_{m+1}^{(+)}}{r \alpha} f_{-}^{(+)}(r)-\frac{e B r}{2} f_{-}^{(+)}(r)=2 M g_{+}^{(+)}(r),
\end{gathered}
$$

with

$$
\begin{gathered}
L_{m}^{(+)}=m-\phi+a M+\frac{1}{2}(1-\alpha), \\
L_{m+1}^{(+)}=m+1-\phi+a M-\frac{1}{2}(1-\alpha) .
\end{gathered}
$$

The solution of the coupled linear differential equation systems (36)-(39) is given by

$$
\begin{gathered}
f_{+}^{(+)}(r)=e^{-e B r^{2} / 4} r^{L_{m}^{(+)} / \alpha}\left[a_{2}+a_{1} M\left(-\frac{e B}{2}\right)^{\Omega_{a}} \Gamma_{a}^{(+)}\right], \\
\mathrm{f}_{-}^{(+)}(r)=e^{e B r^{2} / 4} r^{-L_{m+1}^{(+)} / \alpha}\left[b_{2}-b_{1} M\left(\frac{e B}{2}\right)^{-\Omega_{b}} \Gamma_{b}^{(+)}\right], \\
g_{+}^{(+)}(r)=b_{1} e^{-e B r^{2} / 4} r^{L_{m}^{(+)} / \alpha}, \\
g_{-}^{(+)}(r)=a_{1} e^{e B r^{2} / 4} r^{-L_{m+1}^{(+)} / \alpha},
\end{gathered}
$$

with

$$
\begin{aligned}
& \Omega_{a}=\frac{1}{2 \alpha}\left(L_{m}^{(+)}+L_{m+1}^{(+)}-\alpha\right), \\
& \Omega_{b}=\frac{1}{2 \alpha}\left(L_{m}^{(+)}+L_{m+1}^{(+)}+\alpha\right),
\end{aligned}
$$


where

$$
\begin{aligned}
& \Gamma_{a}^{(+)}=\Gamma\left(-\Omega_{a},-\frac{1}{2} e B r^{2}\right), \\
& \Gamma_{b}^{(+)}=\Gamma\left(\Omega_{b}, \frac{1}{2} e B r^{2}\right),
\end{aligned}
$$

are upper incomplete Gamma functions [96], and $a_{1}, a_{2}, b_{1}$, and $b_{2}$ are constants. Analyzing the solutions (42) and (44), we note that $e^{-e B r^{2} / 4}$ dominates over $r_{m}^{L_{m}^{(+)} / \alpha}$ for any value of $L_{m}^{(+)} / \alpha \geqslant 0$, in such way both solutions converge when $r$ $\longrightarrow r_{\min }$ and $r \longrightarrow \infty$. This will not occur for the function $e^{e B r^{2} / 4}$ in the solutions (43) and (45). Moreover, since the incomplete Gamma functions $\Gamma_{a}^{(+)}$and $\Gamma_{b}^{(+)}$always diverge, then the function $f_{+}^{(+)}(r)$ will only converge when $r \longrightarrow$ $r_{\min }$ if $a_{1}=0$ while the function $f_{-}^{(+)}(r)$ will always diverge when $r \longrightarrow \infty$ and, therefore, will not be a squareintegrable function. Thus, the only allowed solution for the equation systems (36)-(39) results

$$
f_{+}^{(+)}(r)=a_{2} e^{-e B r^{2} / 4} r^{L_{m}^{(+)} / \alpha} \text {, with } \frac{L_{m}^{(+)}}{\alpha} \geqslant 0, r>\frac{a}{\alpha},
$$

with $f_{-}^{(+)}(r)=g_{+}^{(+)}(r)=g_{-}^{(+)}(r)=0$, and the requirement $a_{1}$ $=b_{1}=b_{2}=0$. Solution (49) satisfies Equation (31) and constitutes a bound state solution for the case $E=M$, i.e., an isolated solution to the Dirac equation (4) in the metric spacetime (1). Note there is another particular solution for this case when we reverse the signal of the magnetic field or the charge, i.e., $e B<0$. In this case, the only nonzero component of the normalizable solution $\Psi$ is (Equation (43) with $\left.b_{1}=0\right)$.

$$
f_{-}^{(+)}(r)=b_{2} e^{e B r^{2} / 4} r^{-L_{m+1}^{(+)} / \alpha} \text {, with } \frac{L_{m+1}^{(+)}}{\alpha} \leqslant 0, r>\frac{a}{\alpha} .
$$

Proceeding in an analogous way, now we make $E=-M$ in Equations (28) and (29). We find the system of equations

$$
\begin{gathered}
\frac{d f_{+}^{(-)}(r)}{d r}-\frac{L_{m}^{(-)}}{r \alpha} f_{+}^{(-)}(r)+\frac{e B r}{2} f_{+}^{(-)}(r)=0, \\
\frac{d f_{-}^{(-)}(r)}{d r}+\frac{L_{m+1}^{(-)}}{r \alpha} f_{-}^{(-)}(r)-\frac{e B r}{2} f_{-}^{(-)}(r)=0, \\
\frac{d g_{+}^{(-)}(r)}{d r}-\frac{L_{m}^{(-)}}{r \alpha} g_{+}^{(-)}(r)+\frac{e B r}{2} g_{+}^{(-)}(r)=2 M f_{-}^{(-)}(r), \\
\frac{d g_{-}^{(-)}(r)}{d r}+\frac{L_{m+1}^{(-)}}{r \alpha} g_{-}^{(-)}(r)-\frac{e B r}{2} g_{-}^{(-)}(r)=-2 M f_{+}^{(-)}(r),
\end{gathered}
$$

with

$$
L_{m}^{(-)}=m-\phi-a M+\frac{1}{2}(1-\alpha)
$$

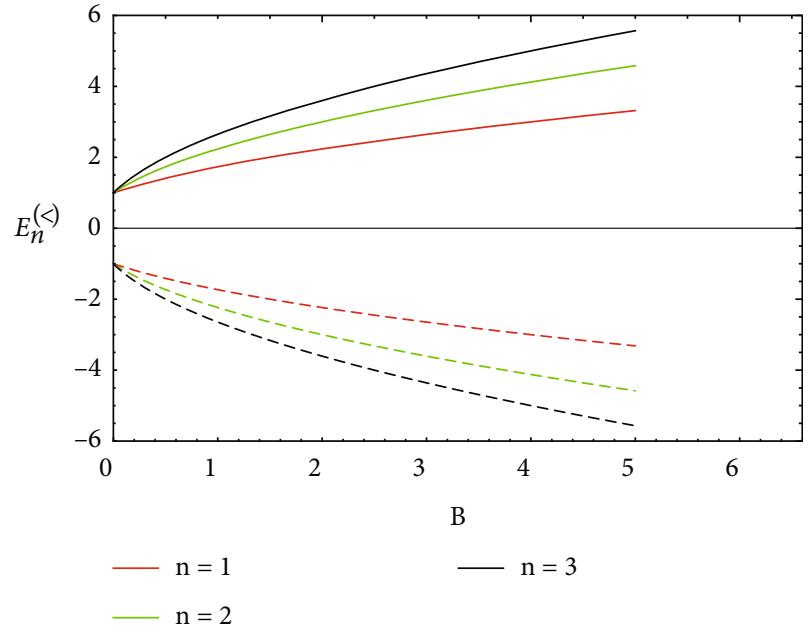

FIgURE 1: Sketch of the energy levels $E_{n}^{(>)}$(Equation (70)) as a function of the magnetic field $B$ for different values of $n$. The positive energies are represented by solid lines and the negative by dashed lines.

$$
L_{m+1}^{(-)}=m+1-\phi-a M-\frac{1}{2}(1-\alpha) .
$$

The solution of the coupled linear ordinary differential equation systems (51)-(54) is given by

$$
\begin{gathered}
f_{+}^{(-)}(r)=c_{1} e^{-e B r^{2} / 4} r^{L_{m}^{(-)} / \alpha}, \\
f_{-}^{(-)}(r)=d_{1} e^{e B r^{2} / 4} r^{-L_{m+1}^{(-)} / \alpha}, \\
g_{+}^{(-)}(r)=e^{-e B r^{2} / 4} r^{L_{m}^{(-)} / \alpha}\left[-d_{1} M\left(-\frac{e B}{2}\right)^{\Lambda_{c}} \Gamma_{c}^{(-)}+d_{2}\right], \\
g_{-}^{(-)}(r)=e^{e B r^{2} / 4} r^{-L_{m+1}^{(-)} / \alpha}\left[c_{1} M\left(\frac{e B}{2}\right)^{-\Lambda_{d}} \Gamma_{d}^{(-)}+c_{2}\right],
\end{gathered}
$$

with

$$
\begin{aligned}
& \Lambda_{c}=\frac{1}{2 \alpha}\left(L_{m}^{(-)}+L_{m+1}^{(-)}-\alpha\right) \\
& \Lambda_{d}=\frac{1}{2 \alpha}\left(L_{m}^{(-)}+L_{\mathrm{m}+1}^{(-)}+\alpha\right)
\end{aligned}
$$

where

$$
\begin{gathered}
\Gamma_{c}^{(-)}=\Gamma\left(-\Lambda_{c},-\frac{1}{2} e B r^{2}\right), \\
\Gamma_{d}^{(-)}=\Gamma\left(\Lambda_{d}, \frac{1}{2} e B r^{2}\right) .
\end{gathered}
$$

By making the same analysis of the solutions as we have made for the case $E=M$, i.e., analyzing the behavior of the functions for $r \longrightarrow \pm \infty$, we find that the only solution that admits bound states is the (59) one. Thus, the appropriate 


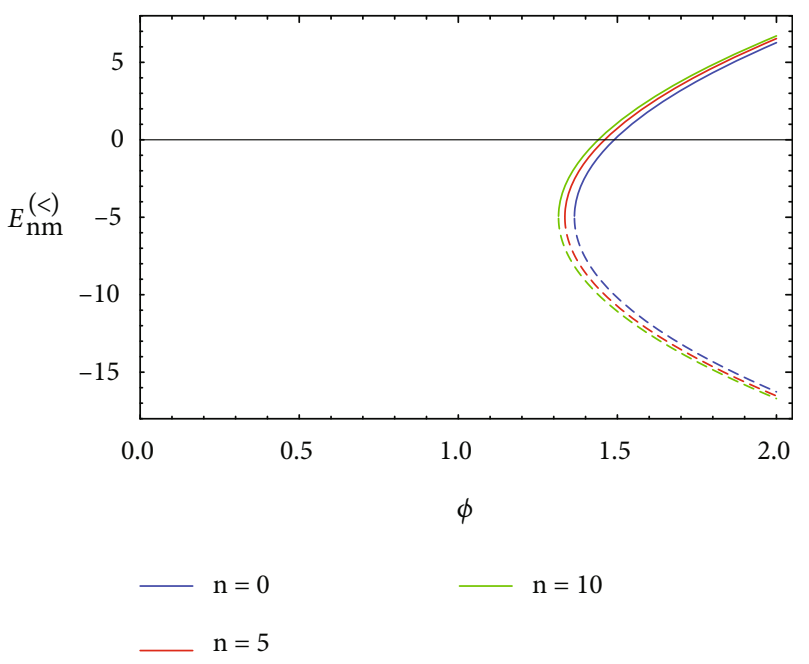

(a)

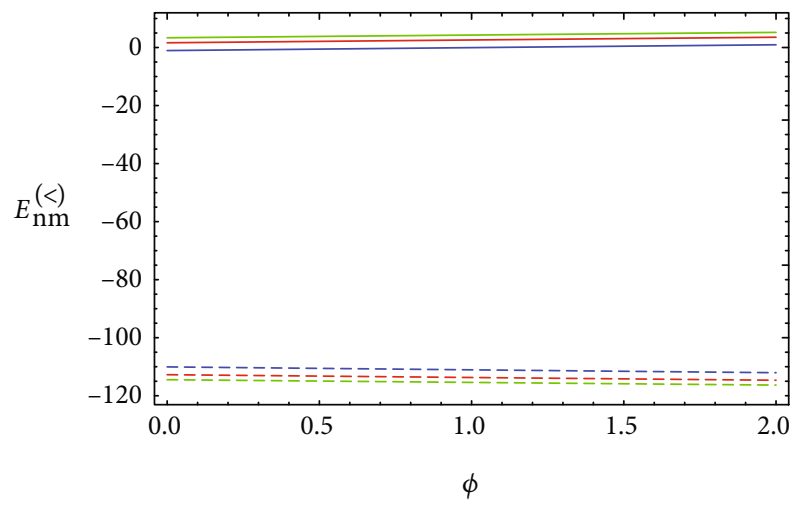

(c)

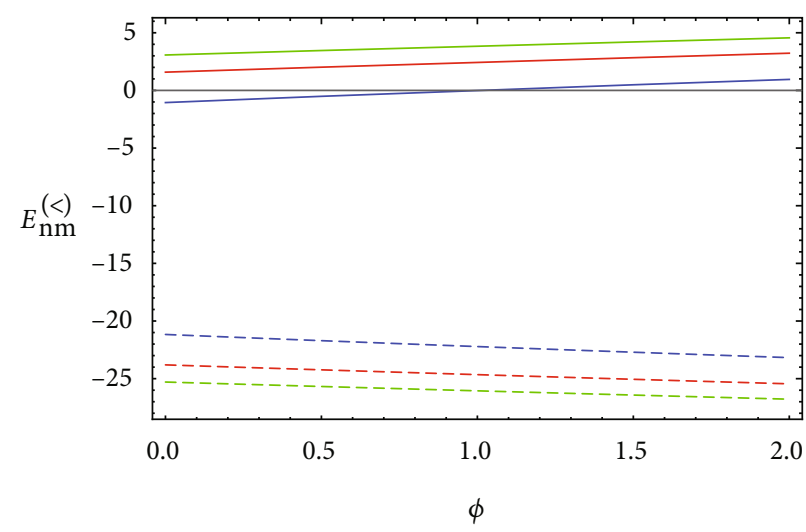

(b)

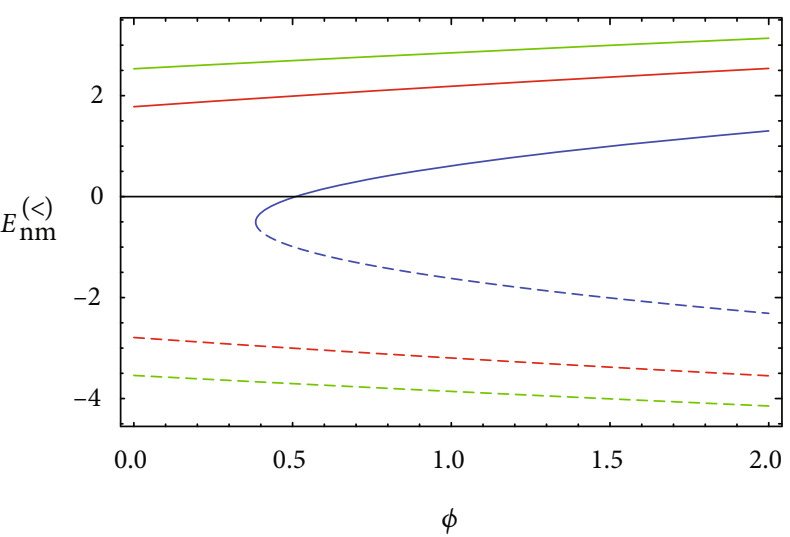

(d)

FIGURE 2: Sketch of the energy (Equation (71)) as a function of $\phi$ for $m=1$. In panel (a), for $B=1.0, a=0.05$ and $\alpha=0.01$. In panel (b), $B=10.0, a=1.0$ and $\alpha=0.9$. In panel (c), $B=50.0, a=1.0$ and $\alpha=0.9$. In panel (d), $B=1.0, a=0.5$ and $\alpha=0.99$. For a particular choice of parameters, ranges of $\phi$ where the energy of a state with a given $n$ is forbidden appear.

solution for the case $E=-M$ satisfying the normalization condition (31) is given by

$$
g_{+}^{(-)}(r)=d_{2} e^{-e B r^{2} / 4} r^{L_{m}^{(-)} / \alpha}, \text { with } \frac{L_{m}^{(-)}}{\alpha} \geqslant 0, r>\frac{a}{\alpha}
$$

with $f_{+}^{(-)}(r)=f_{-}^{(-)}(r)=g_{-}^{(-)}(r)=0$ and $c_{1}=c_{2}=d_{1}=0$. Note that the solutions (49) and (63) are affected by rotation through Equations (40) and (55), respectively. Similarly to the previous case, if $e B<0$, the only nonzero component of the normalizable solution $\Psi$ is (Equation (60) with $c_{1}=0$ )

$$
g_{-}^{(-)}(r)=c_{2} e^{e B r^{2} / 4} r^{-L_{m+1}^{(-)} / \alpha} \text {, with } \frac{L_{m+1}^{(-)}}{\alpha} \leqslant 0, e B<0, r>\frac{a}{\alpha} \text {. }
$$

Moreover, from the discussion of the solutions for each case above, we immediately see that only the first line of the normalization condition (31) is relevant in the current study.

\section{Solution of the Equation of Motion to $E \neq \pm M$}

In this section, we solve the second-order equation to $\psi$ that we find from Equations (28) and (29). The solution of this equation is different from that one calculated in the previous section and allows us to obtain an expression for the particle energies. By isolating $\psi_{2}$ in Equation (29) and replacing in Equation (28), we are able to write the second-order differential equation for $\psi_{1}$ as

$$
\begin{aligned}
\left(E^{2}-M^{2}\right) \psi_{1} & +\partial_{r}^{2} \psi_{1}+\frac{1}{\mathrm{r}} \partial_{r} \psi_{1}-\frac{1}{\alpha r} \sigma^{z} e\left(\partial_{r} A_{\varphi}\right) \psi_{1} \\
& +\frac{1}{\alpha^{2} r^{2}}\left(\partial_{\varphi}+i e A_{\varphi}+i \frac{1-\alpha}{2} \sigma^{z}+i a E\right)^{2} \psi_{1}=0 .
\end{aligned}
$$


Using the decomposition of the fermion field (21) together with Equations (23)-(25), we obtain the radial equation for $f_{+}(r)$

$$
\left(\frac{d^{2}}{d r^{2}}+\frac{1}{r} \frac{d}{d r}-\frac{L_{m}^{2}}{\alpha^{2} r^{2}}-\frac{e^{2} B^{2} r^{2}}{4}+k_{m}^{2}\right) f_{+}(r)=0,
$$

where

$$
\begin{gathered}
k_{m}^{2}=E^{2}-M^{2}+\frac{e B}{\alpha} L_{m}+e B, \\
L_{m}=m-\phi+\frac{(1-\alpha)}{2}+a E .
\end{gathered}
$$

Equation (66) is the confluent hypergeometric equation, and its solution is well known. Thus, it can be shown that the solution to $\psi_{a}$ is

$$
\begin{aligned}
\psi_{a}(r, \varphi)= & a_{n m}\left(\frac{e B}{2}\right)^{\left(1+\left|L_{m}\right| / \alpha\right) / 2} e^{\mathrm{i} m \varphi} r^{\left|L_{m}\right| / \alpha} e^{-e B r^{2} / 4} \\
& \times_{1} F_{1}\left(\frac{1}{2}\left(1+\frac{\left|L_{m}\right|}{\alpha}\right)-\frac{k^{2}}{2 e B}, 1+\frac{\left|L_{m}\right|}{\alpha}, \frac{1}{2} e B r^{2}\right),
\end{aligned}
$$

where ${ }_{1} F_{1}(a, b, z)$ denotes the confluent hypergeometric function of the first kind or Kummer's function $M(a, b, z)$ and $a_{n m}$ the normalization constant. It can be shown that the hypergeometric function ${ }_{1} F_{1}(a, b, z)$ has a divergent behavior for large values of $z$. Because of this, bound state solutions for Equation (69) are only possible if we impose that this function becomes a polynomial of degree $n$. For this to be accomplished, we require that $1 / 2+\left|L_{m}\right| / 2 \alpha-k^{2} / 2 B e$ $=-n$, where $n \in \mathbb{Z}^{*}$, with $\mathbb{Z}^{*}$ denoting the set of the nonnegative integers. However, as we can see in Equation (68), the absolute value of the effective angular moment $L$ is defined in terms of the energy $E$. In this way, to obtain the energy eigenvalues from the above condition, we must consider $L_{m}>0$ and $L_{m}<0$, respectively, and then solve them for $E$. By making this, we get

$$
\begin{gathered}
E_{n}^{(>)}= \pm \sqrt{2 n e B+M^{2}}, \\
E_{n, m}^{(<)}=-\frac{a e B}{\alpha} \pm \frac{1}{\alpha} \sqrt{a^{2} e^{2} B^{2}+\alpha Q_{n, m}}
\end{gathered}
$$

with the following requirement:

$$
a^{2} e^{2} B^{2}+\alpha Q_{n, m} \geqslant 0
$$

where

$$
Q_{n, m}=\alpha e B\left(2 n-\frac{2}{\alpha}\left(m-\phi+\frac{1}{2}\right)+1\right)+\alpha M^{2} .
$$

In Equations (70) and (71), the superscripts $(>,<)$ refer to the energies calculated for $L_{m}>0$ and $L_{m}<0$, respectively.

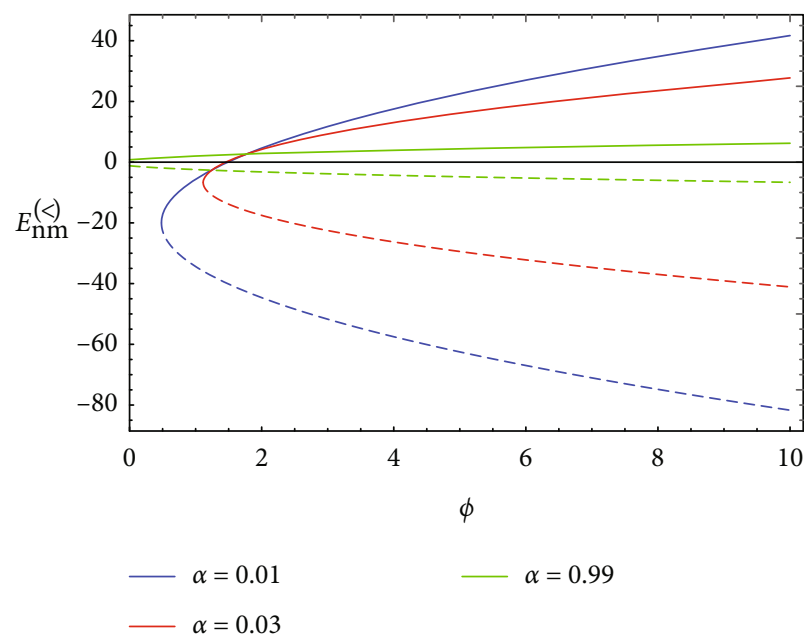

FIGURE 3: Sketch of the energy (Equation (71)) as a function of $\phi$ for $n=1, m=1, B=2, a=0.1$, and different values of $\alpha$. When $\alpha$ approaches 1 , the energy is permissible for all values of $\phi>0$. For small values of $\alpha$, the energy is permissible only from a threshold value of $\phi$. For $\alpha=0.01$ (blue line) and $\alpha=0.03$ (red line), these threshold values are $\phi=0.50$ and $\alpha=1.12$, respectively.

A similar equation for $f_{-}(r)$ can be immediately obtained by making $m \longrightarrow m+1$ and $(1+\alpha) / 2 \longrightarrow-(1+\alpha) / 2$ in Equation (66). We find

$$
\left(\frac{d^{2}}{d r^{2}}+\frac{1}{r} \frac{d}{d r}-\frac{L_{m+1}^{2}}{\alpha^{2} r^{2}}-\frac{e^{2} \mathrm{~B}^{2} r^{2}}{4}+k_{m+1}^{2}\right) f_{-}(r)=0,
$$

where

$$
\begin{gathered}
k_{m+1}^{2}=E^{2}-M^{2}+\frac{e B}{\alpha} L_{m+1}-e B, \\
L_{m+1}=m+1-\phi-\frac{(1-\alpha)}{2}+a E .
\end{gathered}
$$

The function $\psi_{b}(r, \varphi)$ is found by solving Equation (74). The result is

$$
\begin{aligned}
\psi_{b}(r, \varphi)= & a_{n m}\left(\frac{e B}{2}\right)^{\left(1+\left|L_{m+1}\right| / \alpha\right) / 2} e^{i(m+1) \varphi} r^{\left|L_{m+1}\right| / \alpha} e^{-e B r^{2} / 4} \\
& { }_{1} F_{1}\left(\frac{1}{2}+\frac{\left|L_{m+1}\right|}{2 \alpha}+\frac{k_{m+1}^{2}}{2 B e}, 1+\frac{\left|L_{m+1}\right|}{\alpha}, \frac{1}{2} e B r^{2}\right) .
\end{aligned}
$$

The energies obtained from (74) are

$$
\begin{gathered}
E_{n}^{(>)}= \pm \sqrt{2 e B(n+1)+M^{2}}, \\
E_{n, m+1}^{(<)}=-\frac{a e B}{\alpha} \pm \frac{1}{\alpha} \sqrt{a^{2} e^{2} B^{2}+\alpha Q_{n, m+1}},
\end{gathered}
$$




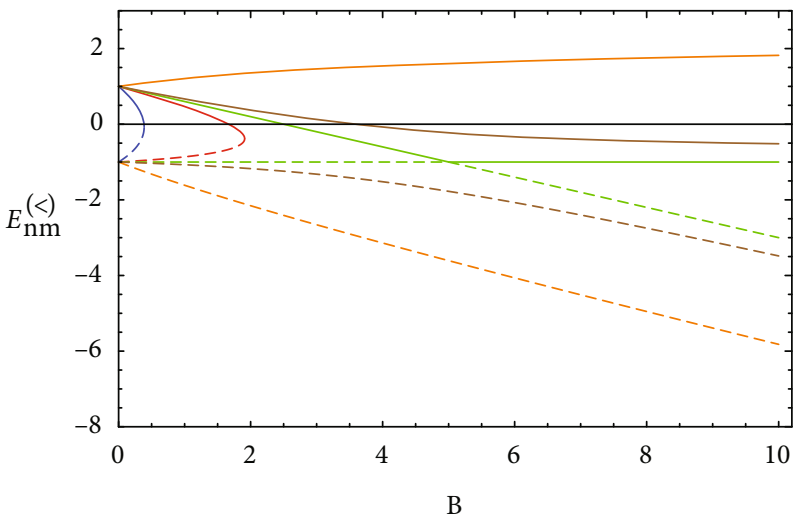

$\begin{aligned}-\phi & =0.10 \\ \phi & =0.60 \\ \varnothing & =0.65\end{aligned}$
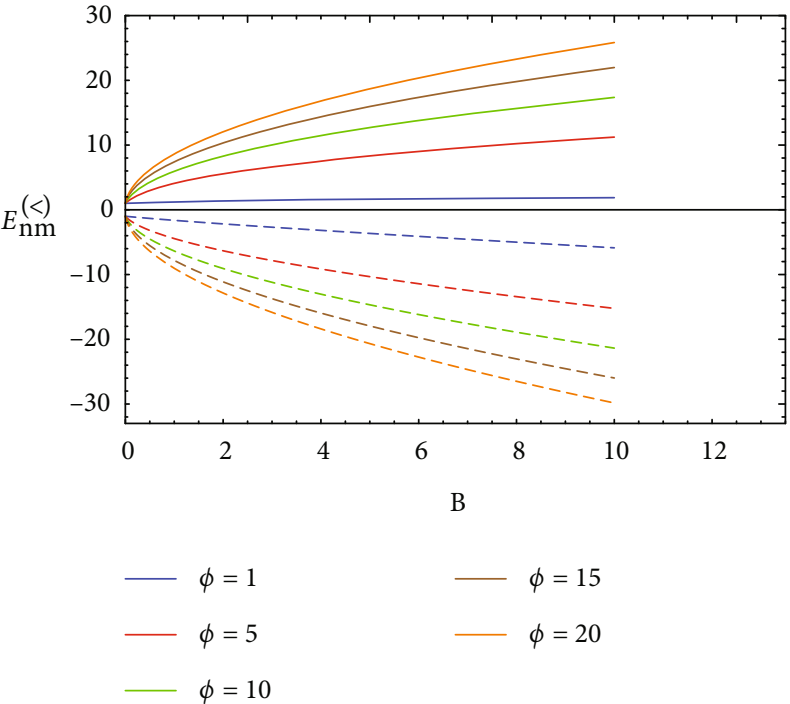

$\phi=15$

$\phi=20$

(b)

Figure 4: Sketch of the energy (Equation (71)) as a function of $B$ for $n=1, m=1, a=0.1$, and $\alpha=0.5$. In panel (a), we consider values of $\phi$ smaller than 1 while in panel (b), we use larger values. The physical implications due to the variation of $\phi$ on the energy spectrum are more evident for values of $\phi$ about 0.65 . For values of $\phi$ larger than 1, the curves assume the same profile.

with the following requirement:

$$
a^{2} e^{2} B^{2}+\alpha Q_{n, m+1} \geqslant 0,
$$

where

$$
Q_{n, m+1}=\alpha e B\left(2 n-\frac{2}{\alpha}\left(m+1-\phi-\frac{1}{2}\right)+1\right)+\alpha M^{2}
$$

Note that two other radial equations can be derived from the equation for $\psi_{2}$ from Equations (28) and (29). However, there is no need to solve them here because they have both energy and wave functions similar to those obtained above. Let us study the energies (70) and (71). First, we see that the energy $E_{n}^{(>)}$depends only on the quantum number $n$ and the magnetic field $B$. If we make $n=0$ in Equation (70), we get $E_{0}^{(>)}= \pm M$. As mentioned above, since we are solving the problem with the requirement that $E \neq \pm M$, then these energies are excluded in the present case.

Now let us investigate the energy levels we find. In all the energy profiles we address here, we use $e=1, M=1$. We also use solid lines to represent the energy of the particle and dashed lines for the energy of the antiparticle. In Figure 1, we show the profile of $E_{n}^{(>)}$for this particular case, where we see clearly that $|E|$ increases when the magnetic field is increased. The energies (70) and (71) denote the relativistic Landau levels in the present context. These energies can be directly compared with those obtained for the relativistic oscillator (Dirac oscillator) addressed in Ref. [77]. Although that scenario is different from the one we are exploring here, there are similarities between the profiles of the energy levels in both models. For example, the energy (47) of the Ref. [77] depends only on the frequency of the oscillator and the quantum number $n$. In our case, by defining the cyclotron frequency $\omega_{c}=e B / M$, Equation (70) results

$$
\tilde{E}_{n}^{(>)}= \pm \sqrt{2 n M \omega_{c}+M^{2}},
$$

which makes such a similarity clear. We can see that the energies (71) and (78) are the only ones that depend on all the physical parameters involved in the current problem. However, it is possible to verify that such spectra are similar. Because of this, we analyze in detail only the energy (71). One of the objectives in our analysis is to show that the combined effect of the external magnetic field, the AharonovBohm flux, the rotation, and the curvature modifies the energy spectrum of the particle. As mentioned in Section 1, one of the new ingredients in the present model is the inclusion of the Aharonov-Bohm flux tube. Then, let us focus on the physical implications of this. We can access varied forms of energy profiles by attributing different values to the parameters of the model. Figure 2 shows the profile of $E_{n, m}^{(<)}$ as a function of $\phi$ by considering four different parameter choices. In these four configurations, we consider $m=1$ and $n=0,5,10$. For $B=1.0, a=0.05$ and $\alpha=0.01$, and the energies are permissible only for values of flux larger than 1.37 (blue line), 1.33 (red line), and 1.30 (green line) (Figure 2(a)). Each value of flux corresponds to the energies $E_{0,1}^{(<)}, E_{5,1}^{(<)}$, and $E_{10,1}^{(<)}$, respectively. The values of $n$ are chosen in such a way the separation between the energies becomes more notable. When this configuration is changed to $B=$ 10.0, $a=1.0$ and $\alpha=0.9$ (Figure 2(b)), the energy levels are more spaced out and a gap between the particle and antiparticle energies emerges. By increasing the magnetic field to $B=50.0$ and maintaining the other parameters, we notice that the antiparticle energy changes faster than the energy 


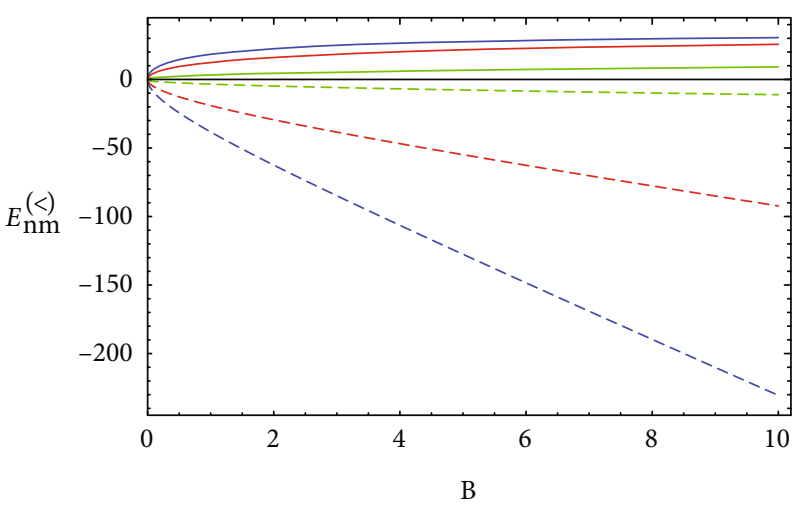

$-\alpha=0.01$

$-\alpha=0.03$

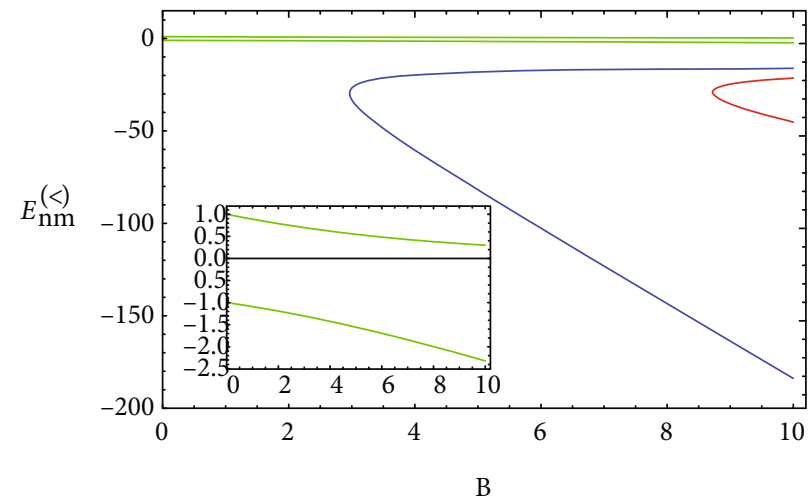

(b)

Figure 5: Sketch of the energy (Equation (71)) as a function of $B$ for $n=1, m=1, a=0.1$, and different values of $\alpha$. In panel (a), $\phi=5$, and in panel (b), $\phi=0$. With the presence of Aharonov-Bohm flux, the energy of the state for a particular value of alpha is permissible for any value of $B$. In the absence of Aharonov-Bohm flux, the energy of a state with a particular value of $\alpha$ is nonpermissible for some values of $B$ (blue and red lines on panel (b)). For ever decreasing $\alpha$ values, the antiparticle energy is more impacted. When $\alpha$ approaches 1 and the flux is zero, the modification in the energy levels is small (see the inset in panel (b)).

of the particle and the spacing between the energy levels decreases (Figure 2(c)). For increasing $B$ values, these effects tend to increase. In the fourth configuration, we use $B=1.0$, $a=0.5$ and $\alpha=0.99$ (Figure 2(d)). When $\alpha$ approaches 1, we can see the energy $E_{0,1}^{(<)}$is only permissible for values of $\phi$ larger than 0.38 while $E_{5,1}^{(<)}$and $E_{10,1}^{(<)}$are defined for any value of $\phi$. In the four settings displayed in Figure 2, we note that $\left|E_{n, m}^{(<)}\right|$increases when $n$ and $\phi$ are increased and the other parameters are kept fixed.

Another interesting configuration is the behavior of $E_{n, m}^{(<)}$ as a function of $\phi$ for $n=1, m=1, B=2.0, a=0.1$, and some values of $\alpha$ (Figure 3). This configuration shows that when $\alpha$ is close to 1 , the energy is defined to all the values of $\phi$ in the considered range. However, for small values of $\alpha$, a range of $\phi$ where the energy is nonpermissible arises. In this process, the most pronounced variations occur in the antiparticle energy.

We can also observe interesting effects in the profile of $E_{n, m}^{(<)}$as a function of $B$. For this case, we consider $n=1, m$ $=1, a=0.1$, and $\alpha=0.5$ and investigate it for two sets of values of $\phi$ as illustrated in Figure 4. By carefully studying this configuration, we can observe several effects for values of $\phi$ smaller than 0.99 (Figure 4(a)). For $\phi=0.10$ (blue line) and $\phi=0.60$ (red line), the energy tends to decrease when $B$ is increased while for $\phi=0.65$ (green line), the energy decreases when $B$ is increased to 5.0. At this value, an overlap between particle and antiparticle energy occurs. The energy increases for values of $B$ higher than 5.0. For $\phi=$ 0.68 (brown line), the energy of the particle tends to decrease when $B$ is increased to 6.0 and then the behavior remains approximately stable. When we look at the energy of the antiparticle, we observe the opposite process. By increasing $B$ to $\phi=4.0$, the energy remains practically stable

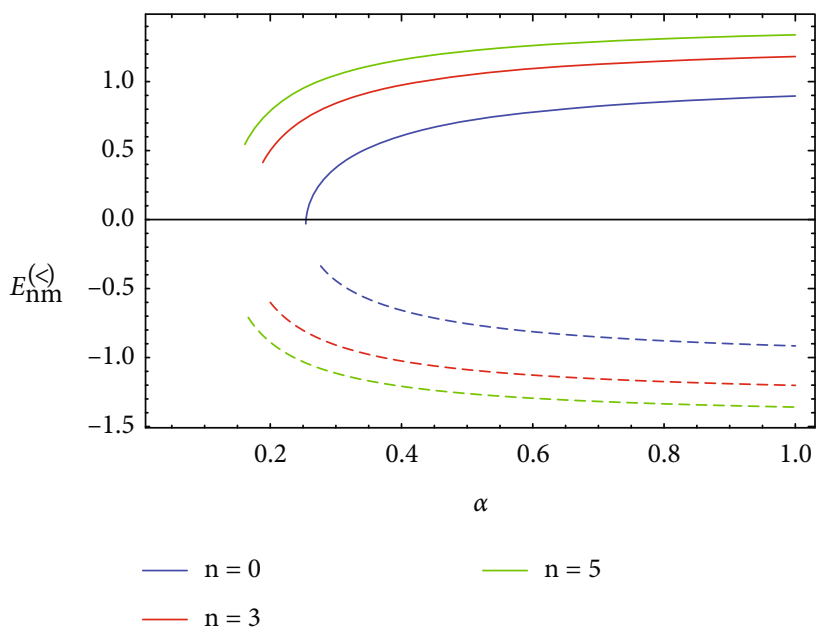

FIGURE 6: Sketch of the energy (Equation (71)) as a function of $\alpha$ for $m=1, B=0.1, a=0.1, \phi=0.1$, and different values of $n$. For values of $\alpha$ smaller than 0.25 , the energy level $E_{0,1}$ (blue line) is nonpermissible. The same occurs with the energy levels $E_{3,1}$ (red line) and $E_{5,1}$ (green line), where they are permissible only for $\alpha$ $>0.19$ and $\alpha>0.16$, respectively.

and then tends to increase as $B$ is increased. When $\phi$ is changed to 0.99 (orange line), both the particle and antiparticle energies increase when $B$ is increased. However, the variation on the antiparticle energy is larger. On the other hand, when we consider different values of $\phi$ higher than 1.0, the profiles are similar (Figure 4(b)). In this case, $\left|E_{n, m}^{(<)}\right|$increases when both $\phi$ and $B$ are increased.

In Figure 5, we investigate the profile of $E_{n, m}^{(<)}$as a function of $B$ for $n=1, m=1, a=0.1$, and different values of $\alpha$ by considering the cases where there is no AharonovBohm flux, and also, considering it is present. In 


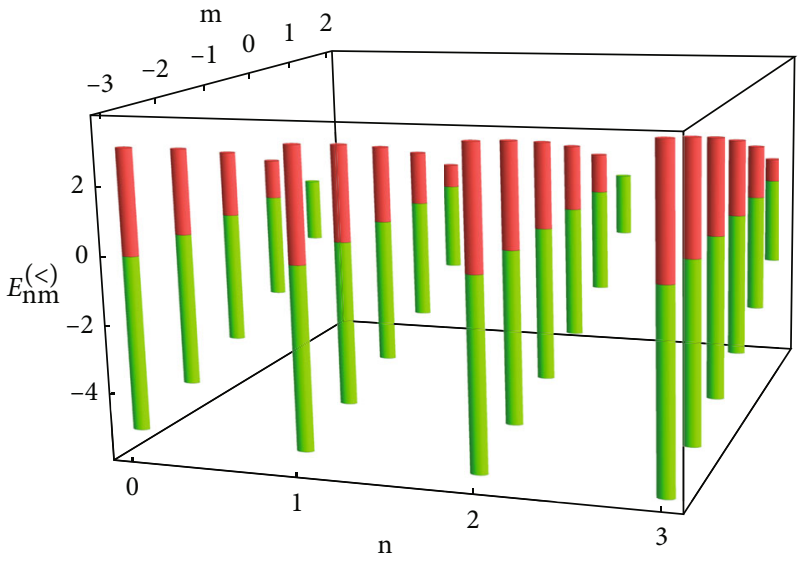

FIGURE 7: Sketch of the energy (Equation (71)) as a function of $n$ and $m$ for $a=0.5, \alpha=0.5, B=1$, and $\phi=1$.

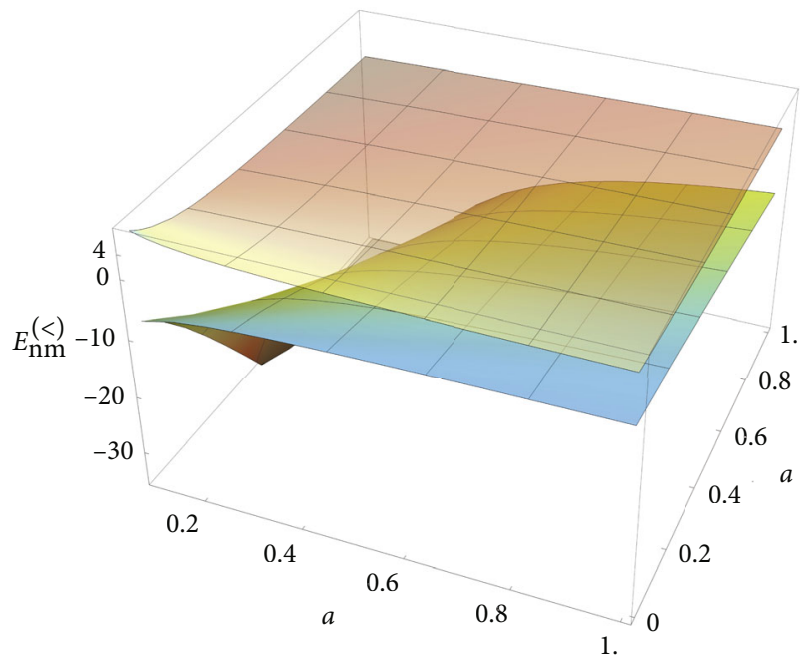

FIGURE 8: Sketch of the energy (Equation (71)) as a function of $\alpha$ and $a$ for $B=4, n=1, \phi=2$, and $m=1$.

Figure 5(a), we consider $\phi=5.0$. We can see that the presence of Aharonov-Bohm flux reveals that the energies are well defined for the considered $\alpha$ values. In the absence of Aharonov-Bohm flux, there are intervals of $B$ associated with nonpermissible energies (Figure 5(b)). For values of $\alpha$ close to 1.0 , the changes in the energies are not too evident, while for smaller values of $\alpha$, the modifications in the energy are more evident, being the antiparticle energy the most affected. In both cases, the energy increases when $B$ increases, and $\alpha$ decreases.

It is also important to examine the energy as a function of $\alpha$, for some values of $n$. Figure 6 illustrates this situation. For $m=1, B=0.1, a=0.1$, and $\phi=0.1$, there are intervals of $\alpha$ leading to forbidden energies. This effect becomes more notable to larger values of $n$. Also, the energy increases when $n$ increases. It is also interesting to explore the energy profiles by performing energy sketches as a function of two quantities of the model. For a given set of fixed parameters, for example, $a=0.5, \alpha=0.5, B=1.0$, and $\phi=1$, we have the

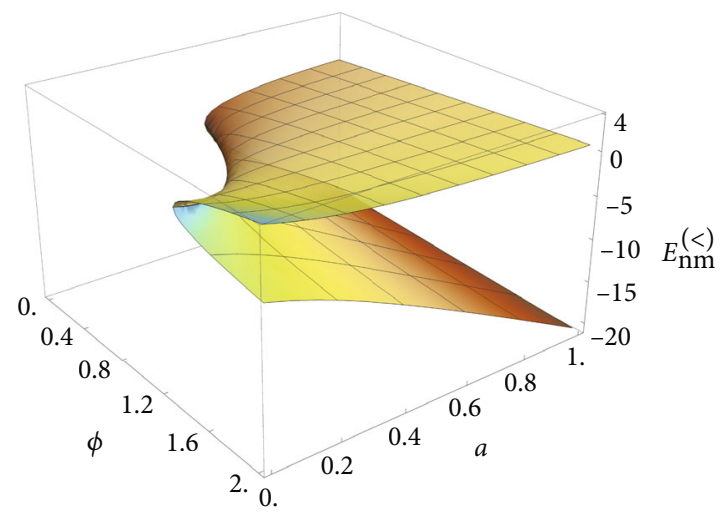

(a)

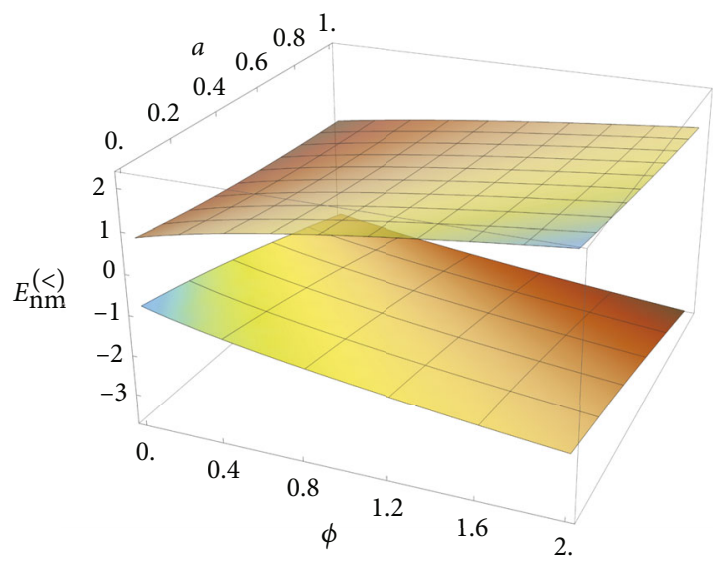

(b)

FIGURE 9: Sketch of the energy (Equation (71)) as a function of $\phi$ and $a$ for $n=1$ and $m=1$. In panel (a), $\alpha=0.1$, and in panel (b), $\alpha=0.9$.

profile of the energy levels as a function of $n$ and $m$ (Figure 7). We can clearly see that $\left|E_{n, m}^{(<)}\right|$increases with $n$ and $m$. The green solid bars denote the discrete energy values for a given $m$ and $n$. On the other hand, when we investigate the behavior of (71) as a function of $\alpha$ and $a$ for specific values of the other parameters, we see that the negative spectrum changes more rapidly when compared with the positive one (Figure 8 ). In the positive spectrum, both rotation and curvature lead to a linear change, except in the region with $\alpha<0.3$ and arbitrary $a$. In the negative spectrum, we see that the curvature effects are more predominant in the region where $\alpha$ has values smaller than 0.2. In this region, any variation in the rotation parameter implies in an abrupt change in the energy spectrum. Modifications in the energies for small values of $\alpha$ are an expected manifestation in our analysis. Its physical implication is inherent in metric (1) and is an immediate consequence of the topological cone, since the curvature becomes larger for smaller values of $\alpha$.

When we study the profile of $E_{n, m}^{(<)}$as a function of $\phi$ and $a$, besides the characteristics displayed in Figures 2 and 3, we also see modifications due to noninertial effects (Figure 9). For this particular case, we see that for small values of $\phi$ 


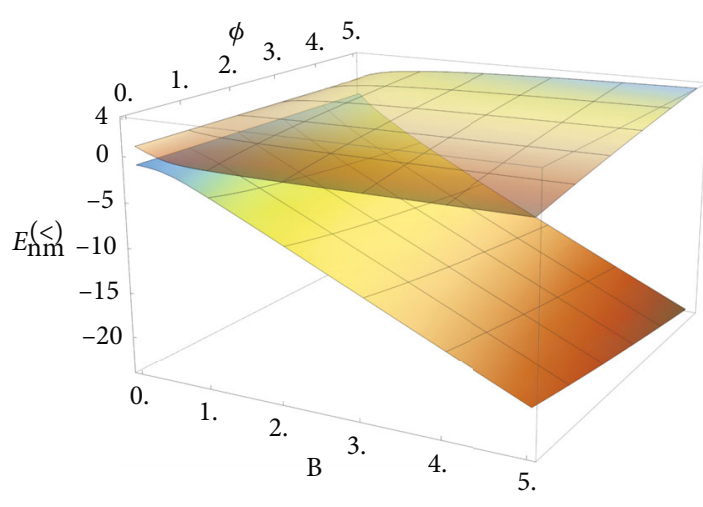

FIGURE 10: Sketch of the energy (Equation (71)) as a function of $B$ and $\phi$ for $a=1, \alpha=0.5, m=1$, and $n=1$.

and $a$, some energy levels are forbidden. This characteristic is observed for $n=1, m=1$, and $\alpha=0.1$ (Figure 9(a)). By changing $\alpha$ to 0.9 , this effect disappears, and the energies are set to all values of $\phi$ and $a$ in the considered range (Figure 9(b)).

To complete our analysis, we investigate the profile of the energy (71) as a function of magnetic field $B$ and the magnetic flux through the solenoid, $\phi$ (Figure 10). Similarly to Figure 8 , by fixing the other parameters, we see that the energy of the antiparticle varies more rapidly when compared to the energy of the particle (Figure 10). Clearly, we observe that the energy of the particle varies very slowly throughout the region of flux and magnetic field. As a final commentary, we clarify that the cases discussed in Figures 7,8 , and 10 can be investigated for other fixed parameter values. In this way, it can be shown that there are forbidden energies, depending on the values of the parameters considered. In general, this occurs when both the $\alpha$ parameter and the rotation parameter $a$ are smaller than 0.3 and the other parameters assuming higher values than those we use here.

\section{Conclusions}

In the present manuscript, we have addressed the problem of the relativistic quantum motion of an electron in the spinning cosmic string background considering the presence of a uniform magnetic field and the Aharonov-Bohm potential. We have shown that this combination of potentials allows bound state configurations in the scenario of first-order solutions as well as in the case of second-order solutions of the Dirac equation. It is worth noting the role played by the two different terms in the vector potential. As already known in the literature, we have shown that the uniform field is responsible for a behavior analog to a harmonic oscillator, which leads to the relativistic Landau quantization. Also, we saw the Aharonov-Bohm flux contributes to the angular momentum and energy spectrum of the particle. In the case of first-order solutions, which were obtained by solving Equations (49) and (63) for $E=+M$ and $E=-M$, respectively, the oscillator-like behavior provided by the uniform magnetic field guarantees the convergent first-order solutions and, consequently, the existence of bound states.
The isolated solutions obtained (Equations (49) and (63)) are particular solutions of the Dirac equation (4).

We have also studied the more general problem by solving the second-order equation implied by Equations (28) and (29) for the upper component of the Dirac spinor for $E \neq \pm M$. Using appropriate solutions (Equation (20)), we have derived the radial equation and shown that its solution depends on the Kummer functions. From these functions, we have extracted the expression for the energy levels of the particle (Equations (70) and (71)). For the field configuration considered, we have found that the effective angular momentum of the electron depends on its energy and the Aharonov-Bohm flux tube. Besides, the potential vector corresponding to the uniform field leads to a charged oscillator. Thus, such field superposition provides distinct effects on the motion of the particle. Additionally, in some cases, the rotation produces a combined effect with both the uniform magnetic field and the curvature (see Equation (71)). We have shown that the energy levels of the particle and antiparticle depend on the values of the physical parameters involved. In the case of energy (71), its validity is conditioned to Equation (72). Depending on the choice we make for the parameters, we can obtain forbidden energies. Sketches in Figures 7, 8, and 10 illustrate the profiles of the particle and antiparticle energies and show that they belong to the same spectrum. The effects of curvature and rotation are more evident when $\alpha<0.3$, being the antiparticle energy the most affected. Summarizing our results, we can state that the rotation breaks the symmetry in the energies (71) and (78) about $E_{n, m}^{(<)}=0$ and $E_{n, m+1}^{(<)}=0$, respectively, while the energies (70) and (77) are symmetrical. When $a \longrightarrow 0$ or $B$ $\longrightarrow 0$ in (71) and (78), this symmetry tends to be recovered. In the case of a strong magnetic field, the separation between the particle and antiparticle energies increases in comparison with the case of a weak field. When $a$ is large and $\alpha$ $\longrightarrow 0$, the energies increase rapidly. We saw also that the inclusion of the Aharonov-Bohm flux can produce notable modifications in the energy profile. For instance, it can affect even the behavior of the energy as a function of the uniform magnetic field. As a final comment, we would like to emphasize that the model studied in this article generalizes others found in the literature, such as those of Refs. $[68,72]$ for the case including a superposition of external magnetic fields and the investigation of isolated solutions of the Dirac equation. Furthermore, we present a detailed discussion on the energy levels of the particle which, in general, is not found in the literature.

\section{Data Availability}

No data were used to support this study.

\section{Disclosure}

A previous version of this manuscript was presented in arXiv:2006.06511. 


\section{Conflicts of Interest}

The authors declare that they have no conflicts of interest.

\section{Acknowledgments}

We would like to thank E.R.B. Mello (Universidade Federal da Paraíba, PB, Brazil) for his remarks and comments. This work was partially supported by the Brazilian agencies CAPES, CNPq, and FAPEMA. EOS acknowledges CNPq Grant 307203/2019-0 and FAPEMA Grant 01852/14. This study was financed in part by the Coordenação de Aperfeiçoamento de Pessoal de Nvel Superior-Brasil (CAPES)Finance Code 001. MMC acknowledges CAPES Grant 88887.358036/2019-00.

\section{References}

[1] C. Lanczos, The Variational Principles of Mechanics, Courier Corporation, 2012.

[2] J. J. Sakurai and J. Napolitano, Modern Quantum Mechanics, Pearson Harlow, 2014.

[3] I. Marvian, "Symmetry-protected topological entanglement," Physical Review B, vol. 95, no. 4, article 045111, 2017.

[4] H. Westman and S. Sonego, "Coordinates, observables and symmetry in relativity," Annals of Physics, vol. 324, no. 8, pp. 1585-1611, 2009.

[5] W. M. Gibson and B. R. Pollard, Symmetry Principles Particle Physics, CUP Archive, 1980.

[6] J. Preskill, S. P. Trivedi, F. Wilczek, and M. B. Wise, "Cosmology and broken discrete symmetry," Nuclear Physics B, vol. 363 , no. 1, pp. 207-220, 1991.

[7] H. Ikegami, Y. Tsutsumi, and K. Kono, "Chiral symmetry breaking in Superfluid3He-A," Science, vol. 341, no. 6141, pp. 59-62, 2013.

[8] J. Harada, "Spontaneous symmetry breaking in superfluid helium-4," Physics Letters A, vol. 367, no. 6, pp. 489-492, 2007.

[9] K. Baumann, R. Mottl, F. Brennecke, and T. Esslinger, "Exploring symmetry breaking at the Dicke quantum phase transition," Physical Review Letters, vol. 107, no. 14, article 140402, 2011.

[10] M. Kleman and J. Friedel, "Disclinations, dislocations, and continuous defects: a reappraisal," Reviews of Modern Physics, vol. 80 , no. 1 , pp. $61-115,2008$.

[11] R. A. Puntigam and H. H. Soleng, "Volterra distortions, spinning strings, and cosmic defects," Classical and Quantum Gravity, vol. 14, no. 5, pp. 1129-1149, 1997.

[12] J. Sólyom, Fundamentals of the Physics of Solids: Volume 1: Structure and Dynamics, Springer Science \& Business Media, 2007.

[13] O. D. Lavrentovich, P. Pasini, C. Zannoni, and S. Zumer, Defects in Liquid Crystals: Computer Simulations, Theory and Experiments, Springer Science \& Business Media, 2012.

[14] J. S. Alden, A. W. Tsen, P. Y. Huang et al., "Strain solitons and topological defects in bilayer graphene," Proceedings of the National Academy of Sciences of the United States of America, vol. 110, no. 28, pp. 11256-11260, 2013.

[15] K. Bakke, A. Y. Petrov, and C. Furtado, "A Kaluza-Klein description of geometric phases in graphene," Annals of Physics, vol. 327, no. 12, pp. 2946-2954, 2012.
[16] L. Kou, C. Tang, W. Guo, and C. Chen, "Tunable magnetism in strained graphene with topological line defect," ACS Nano, vol. 5, no. 2, pp. 1012-1017, 2011.

[17] R. Durrer, "Topological defects in cosmology," New Astronomy Reviews, vol. 43, no. 2-4, pp. 111-156, 1999.

[18] K. Kawaguchi, R. Kageyama, and M. Sano, "Topological defects control collective dynamics in neural progenitor cell cultures," Nature, vol. 545, no. 7654, pp. 327-331, 2017.

[19] T. B. Saw, A. Doostmohammadi, V. Nier et al., "Topological defects in epithelia govern cell death and extrusion," Nature, vol. 544, no. 7649, pp. 212-216, 2017.

[20] M. B. Hindmarsh and T. W. B. Kibble, "Cosmic strings," Reports on Progress in Physics, vol. 58, no. 5, pp. 477-562, 1995.

[21] A. Vilenkin and E. P. S. Shellard, Cosmic Strings and Other Topological Defects, Cambridge University Press, 2000.

[22] F. Moraes, "Condensed matter physics as a laboratory for gravitation and cosmology," Brazilian Journal of Physics, vol. 30, no. 2, p. 304, 2000.

[23] W. B. Thomas, "Topology of cosmic domains and strings," Journal of Physics A: Mathematical and General, vol. 9, no. 8, pp. 1387-1398, 1976.

[24] G. D. A. Marques and V. B. Bezerra, "Hydrogen atom in the gravitational fields of topological defects," Physical Review D, vol. 66, no. 10, article 105011, 2002.

[25] G. D. A. Marques, V. B. Bezerra, and S. G. Fernandes, "Exact solution of the Dirac equation for a Coulomb and scalar potentials in the gravitational field of a cosmic string," Physics Letters A, vol. 341, no. 1-4, pp. 39-47, 2005.

[26] E. R. Bezerra de Mello, V. B. Bezerra, A. A. Saharian, and A. S. Tarloyan, "Vacuum polarization induced by a cylindrical boundary in the cosmic string spacetime," Physical Review D, vol. 74, no. 2, article 025017, 2006.

[27] J. Carvalho, C. Furtado, and F. Moraes, "Dirac oscillator interacting with a topological defect," Physical Review A, vol. 84, no. 3, article 032109, 2011.

[28] F. M. Andrade and E. O. Silva, "Effects of spin on the dynamics of the 2D Dirac oscillator in the magnetic cosmic string background," European Physical Journal C: Particles and Fields, vol. 74, no. 12, p. 3187, 2014.

[29] Y. Yang, S.-H. Cai, Z.-W. Long, H. Chen, and C.-Y. Long, "Exact solution of the $(1+2)$-dimensional generalized Kemmer oscillator in the cosmic string background with the magnetic field," Chinese Physics B, vol. 29, no. 7, article 070302, 2020.

[30] N. Messai and A. Boumali, "Exact solutions of a twodimensional Kemmer oscillator in the gravitational field of cosmic string," The European Physical Journal Plus, vol. 130, no. 7, p. 140, 2015.

[31] K. Bakke, C. Furtado, and H. Belich, "Relativistic Anandan quantum phase and the Aharonov-Casher effect under Lorentz symmetry breaking effects in the cosmic string spacetime," Annals of Physics, vol. 372, pp. 544-552, 2016.

[32] E. R. Bezerra de Mello, A. A. Saharian, and A. Mohammadi, "Induced fermionic current by a magnetic flux in a cosmic string spacetime at finite temperature," International Journal of Modern Physics A, vol. 31, article 1641021, 2016.

[33] D. J. Griffiths, Introduction to Quantum Mechanics, AddisonWesley, 2nd edition, 2005.

[34] Y. Aharonov and D. Bohm, "Significance of electromagnetic potentials in the quantum theory," Physics Review, vol. 115, no. 3, pp. 485-491, 1959.

[35] M. Peshkin, The Aharonov-Bohm Effect, Springer, 1989. 
[36] W. C. F. da Silva and K. Bakke, "Non-relativistic effects on the interaction of a point charge with a uniform magnetic field in the distortion of a vertical line into a vertical spiral spacetime," Classical and Quantum Gravity, vol. 36, no. 23, article 235002, 2019.

[37] G. d. A. Marques, C. Furtado, V. B. Bezerra, and F. Moraes, "Landau levels in the presence of topological defects," Journal of Physics A: Mathematical and General, vol. 34, no. 30, pp. 5945-5954, 2001.

[38] S. Azevedo and F. Moraes, "Topological Aharonov-Bohm effect around a disclination," Physics Letters A, vol. 246, no. 3-4, pp. 374-376, 1998.

[39] H. Belich, E. O. Silva, J. Ferreira, and M. T. D. Orlando, "Aharonov-Bohm-Casher problem with a nonminimal Lorentzviolating coupling," Phys. Rev. D, vol. 83, no. 12, article 125025, 2011.

[40] E. O. Silva, S. C. Ulhoa, F. M. Andrade, C. Filgueiras, and R. G. G. Amorim, "Quantum motion of a point particle in the presence of the Aharonov-Bohm potential in curved space," Annals of Physics, vol. 362, pp. 739-751, 2015.

[41] V. Salem, R. F. Costa, E. O. Silva, and F. M. Andrade, "Selfadjoint extension approach for singular Hamiltonians in $(2+$ 1) dimensions," Frontiers in Physics, vol. 7, p. 175, 2019.

[42] Houcine Aounallah and Abdelmalek Boumali, "Solutions of the Duffin-Kemmer equation in non-commutative space of cosmic string and magnetic monopole with allowance for the Aharonov-Bohm and Coulomb potentials," Physics of Particles and Nuclei Letters, vol. 16, no. 3, pp. 195-205, 2019.

[43] A. Boumali and H. Aounallah, "Exact solutions of scalar bosons in the presence of the Aharonov-Bohm and Coulomb potentials in the gravitational field of topological defects," Advances in High Energy Physics, vol. 2018, Article ID 1031763, 9 pages, 2018.

[44] J. Oliveira, G. Garcia, C. Furtado, and S. Sergeenkov, "Evolution of Landau levels in graphene-based topological insulators in the presence of wedge disclinations," Annals of Physics, vol. 383, pp. 610-619, 2017.

[45] J. Amaro Neto, J. R. D. S. Oliveira, C. Furtado, and S. Sergeenkov, "Quantum ring in gapped graphene layer with wedge disclination in the presence of a uniform magnetic field," The European Physical Journal Plus, vol. 133, no. 5, p. $185,2018$.

[46] R. L. L. Vitoria and K. Bakke, "Aharonov-Bohm effect for bound states in relativistic scalar particle systems in a spacetime with a spacelike dislocation," International Journal of Modern Physics D, vol. 27, no. 2, article 1850005, 2018.

[47] E. R. Figueiredo Medeiros and E. R. Bezerra de Mello, "Relativistic quantum dynamics of a charged particle in cosmic string spacetime in the presence of magnetic field and scalar potential," The European Physical Journal C, vol. 72, no. 6, p. 2051, 2012.

[48] M. S. Maior de Sousa, R. F. Ribeiro, and E. R. Bezerra de Mello, "Induced fermionic current by a magnetic tube in the cosmic string spacetime," Physical Review D, vol. 93, no. 4, article 043545, 2016.

[49] J. T. Giblin, J. B. Mertens, G. D. Starkman, and C. Tian, "Cosmic expansion from spinning black holes," Classical and Quantum Gravity, vol. 36, no. 19, article 195009, 2019.

[50] V. A. Korotky, E. Masár, and Y. N. Obukhov, "In the quest for cosmic rotation," Universe, vol. 6, no. 1, p. 14, 2020.
[51] K. Bakke, V. B. Bezerra, and R. L. L. Vitoria, "Scalar field in a uniformly rotating frame in the time-dislocation space-time," International Journal of Modern Physics A, vol. 35, no. 22, article 2050129, 2020.

[52] Z. Wang, Z.-w. Long, C.-y. Long, and M.-l. Wu, "Relativistic quantum dynamics of a spinless particle in the SomRaychaudhuri spacetime," The European Physical Journal Plus, vol. 130, no. 3, p. 1, 2015.

[53] E. O. Silva, "Ground state of a bosonic massive charged particle in the presence of external fields in a Gödel-type spacetime," The European Physical Journal Plus, vol. 133, no. 12, p. 1, 2018.

[54] Y. Aharonov and G. Carmi, "Quantum aspects of the equivalence principle," Foundations of Physics, vol. 3, no. 4, pp. 493-498, 1973.

[55] M. D. Semon, "Experimental verification of an AharonovBohm effect in rotating reference frames," Foundations of Physics, vol. 12, no. 1, pp. 49-57, 1982.

[56] B. L. Johnson, "Inertial forces and the Hall effect," American Journal of Physics, vol. 68, no. 7, pp. 649-653, 2000.

[57] M. Matsuo, J.'i. Ieda, E. Saitoh, and S. Maekawa, "Spin-dependent inertial force and spin current in accelerating systems," Physical Review B, vol. 84, no. 10, article 104410, 2011.

[58] D. Chowdhury and B. Basu, "Spin transport in non-inertial frame," Physica B: Condensed Matter, vol. 448, pp. 155-161, 2014.

[59] G. Q. Garcia, E. Cavalcante, A. M. D. M. Carvalho, and C. Furtado, "The geometric theory of defects description for C60 fullerenes in a rotating frame," The European Physical Journal Plus, vol. 132, no. 4, p. 183, 2017.

[60] M. Ono, H. Chudo, K. Harii et al., "Barnett effect in paramagnetic states," Physical Review B, vol. 92, no. 17, article 174424, 2015.

[61] N. Zettili and Q. Mechanics, Concepts and Applications, John Wiley and Sons, 2009.

[62] A. Danner, B. Demirel, W. Kersten et al., "Spin-rotation coupling observed in neutron interferometry," NPJ Quantum Information, vol. 6, no. 1, 2020.

[63] G. Clement, "Rotating string sources in three-dimensional gravity," Annals of Physics, vol. 201, no. 2, pp. 241-257, 1990.

[64] C. Muniz, V. Bezerra, and M. Cunha, "Landau quantization in the spinning cosmic string spacetime," Annals of Physics, vol. 350, pp. 105-111, 2014.

[65] H. Hassanabadi, A. Afshardoost, and S. Zarrinkamar, "On the motion of a quantum particle in the spinning cosmic string space-time," Annals of Physics, vol. 356, pp. 346-351, 2015.

[66] K. Bakke and C. Furtado, "Bound states for neutral particles in a rotating frame in the cosmic string spacetime," Physical Review D, vol. 82, no. 8, article 084025, 2010.

[67] K. Bakke, "On the rotating effects and the Landau-AharonovCasher system subject to a hard-wall confining potential in the cosmic string spacetime," International Journal of Theoretical Physics, vol. 54, no. 7, pp. 2119-2126, 2015.

[68] R. R. S. Oliveira, "Topological and noninertial effects in an Aharonov-Bohm ring," General Relativity and Gravitation, vol. 51, no. 9, p. 120, 2019.

[69] Z. Wang, Z.-w. Long, C.-y. Long, and B.-q. Wang, "The study of a spinless relativistic particle in the spinning cosmic string space-time," Canadian Journal of Physics, vol. 95, no. 4, pp. 331-335, 2017.

[70] K. Bakke and C. Furtado, "On the interaction of the Dirac oscillator with the Aharonov-Casher system in topological 
defect backgrounds," Annals of Physics, vol. 336, pp. 489-504, 2013.

[71] R. Oliveira, "Topological, noninertial and spin effects on the 2D Dirac oscillator in the presence of the Aharonov-Casher effect," The European Physical Journal C, vol. 79, no. 9, p. $725,2019$.

[72] B.-Q. Wang, Z.-W. Long, C.-Y. Long, and S.-R. Wu, "The study of a half-spin relativistic particle in the rotating cosmic string space-time," International Journal of Modern Physics A, vol. 33, no. 27, article 1850158, 2018.

[73] M. Peshkin, "Aharonov-Bohm effect in bound states: theoretical and experimental status," Physical Review A, vol. 23, no. 1, pp. 360-361, 1981.

[74] Y. Yang, Z.-W. Long, H. Chen, Z.-L. Zhao, and C.-Y. Long, "Aharonov-Bohm effect on the generalized Duffin-Kemmer-Petiau oscillator in the Som-Raychaudhuri spacetime," Modern Physics Letters A, vol. 36, no. 9, article 2150059, 2021.

[75] H. Chen, Z. W. Long, Q. K. Ran, Y. Yang, and C. Y. Long, "Aharonov-Bohm effect on the generalized Dirac oscillator in a cosmic dislocation space-time," Europhysics Letters, vol. 132, article 50006, 2020.

[76] F. Ahmed, "Aharonov-Bohm effect for bound states on spin-0 massive charged particles in a Gödel-type space-time with Coulomb potential," Communications in Theoretical Physics, vol. 72, no. 7, article 075102, 2020.

[77] M. Hosseinpour, H. Hassanabadi, and M. de Montigny, "The Dirac oscillator in a spinning cosmic string spacetime," The European Physical Journal C, vol. 79, no. 4, p. 311, 2019.

[78] M. M. Cunha, H. S. Dias, and E. O. Silva, "Dirac oscillator in a spinning cosmic string spacetime in external magnetic fields: investigation of the energy spectrum and the connection with condensed matter physics," Physical Review D, vol. 102, no. 10, article 105020, 2020.

[79] P. O. Mazur, "Spinning cosmic strings and quantization of energy," Physical Review Letters, vol. 57, no. 8, pp. 929-932, 1986.

[80] S. Deser, R. Jackiw, and G. 't Hooft, "Physical cosmic strings do not generate closed timelike curves," Physical Review Letters, vol. 68, no. 3, pp. 267-269, 1992.

[81] B. Jensen and H. H. Soleng, "General-relativistic model of a spinning cosmic string," Physical Review D, vol. 45, no. 10, pp. 3528-3533, 1992.

[82] M. Ringbauer, M. A. Broome, C. R. Myers, A. G. White, and T. C. Ralph, "Experimental simulation of closed timelike curves," Nature Communications, vol. 5, no. 1, p. 1, 2014.

[83] F. Lobo and P. Crawford, "Time, Closed Timelike Curves and Causality," in The Nature of Time: Geometry, Physics and Perception, R. Buccheri, M. Saniga, and W. M. Stuckey, Eds., pp. 289-296, Springer, 2003.

[84] G. Martín-Vázquez and C. Sabín, "Closed timelike curves and chronology protection in quantum and classical simulators," Classical and Quantum Gravity, vol. 37, no. 4, article 045013, 2020.

[85] F. Cooperstock and S. Tieu, "Closed timelike curves and time travel: dispelling the myth," Foundations of Physics, vol. 35, no. 9, pp. 1497-1509, 2005.

[86] T. C. Ralph and C. R. Myers, "Information flow of quantum states interacting with closed timelike curves," Physical Review $A$, vol. 82, no. 6, article 062330, 2010.

[87] C. R. Hagen, "Aharonov-Bohm scattering of particles with spin," Physical Review Letters, vol. 64, no. 5, pp. 503-506, 1990.
[88] D. K. Park and Jae Geun Oh, "Self-adjoint extension approach to the spin-1/2 Aharonov-Bohm-Coulomb problem," Physical Review D, vol. 50, no. 12, pp. 7715-7720, 1994.

[89] F. M. Andrade, E. O. Silva, and M. Pereira, "Physical regularization for the spin-1/2Aharonov-Bohm problem in conical space," Physical Review D, vol. 85, no. 4, article 041701, 2012.

[90] F. M. Andrade, E. O. Silva, and M. Pereira, "On the spin- 1/2 Aharonov-Bohm problem in conical space: bound states, scattering and helicity nonconservation," Annals of Physics, vol. 339, pp. 510-530, 2013.

[91] D. F. Lima, F. M. Andrade, L. B. Castro, C. Filgueiras, and E. O. Silva, "On the 2D Dirac oscillator in the presence of vector and scalar potentials in the cosmic string spacetime in the context of spin and pseudospin symmetries," The European Physical Journal C, vol. 79, no. 7, p. 596, 2019.

[92] F. M. Andrade and E. O. Silva, "Remarks on the Dirac oscillator in $(2+1)$ dimensions," EPL, vol. 108, no. 3, p. 30003, 2014.

[93] L. B. Castro and A. S. de Castro, "Missing solution in a Cornell potential," Annals of Physics, vol. 338, pp. 278-282, 2013.

[94] L. B. Castro and A. S. de Castro, "Relativistic confinement of neutral fermions with a trigonometric tangent potential," Journal of Physics A: Mathematical and Theoretical, vol. 40, no. 2, pp. 263-270, 2007.

[95] A. S. de Castro and M. Hott, "Trapping neutral fermions with kink-like potentials," Physics Letters A, vol. 351, no. 6, pp. 379383, 2006.

[96] M. Abramowitz and I. A. Stegun, Eds., Handbook of Mathematical Functions, Dover Publications, New York, NY, USA, 1972. 